\title{
An effective black hole remnant via infinite evaporation time due to generalized uncertainty principle
}

\author{
Yen Chin Ong \\ Center for Gravitation and Cosmology, College of Physical Science and Technology, \\ Yangzhou University, \\ 180 Siwangting Road, Yangzhou City 225002, Jiangsu Province, P.R. China \\ School of Physics and Astronomy, Shanghai Jiao Tong University, \\ 800 Dongchuan Road, Minhang 200240, Shanghai, P.R. China \\ E-mail: ycong@yzu.edu.cn
}

ABSTRACT: We study a form of generalized uncertainty principle (GUP) that leads to vanishing quantum effect, i.e. $\Delta x \Delta p \sim 0$, at sufficiently high momenta. We find that such a GUP allows black holes to evaporate completely, however this process takes an infinite amount of time to achieve, resulting in a metastable remnant. We also discuss some connections between the proposed generalized uncertainty principle with other quantum gravity models.

KeYwords: Black Holes, Models of Quantum Gravity

ArXiv EPrint: 1806.03691 


\section{Contents}

1 Generalized uncertainty principle: positive vs. negative correction 1

2 The lifetime of GUP-corrected black holes 3

3 Sparsity makes lifetime even longer 5

4 Discussion: the final fate of GUP-corrected black holes 7

\section{Generalized uncertainty principle: positive vs. negative correction}

The generalized uncertainty principle (GUP) is a quantum gravity inspired correction to the Heisenberg's uncertainty principle. The simplest form of GUP is

$$
\Delta x \Delta p \geqslant \frac{1}{2}\left[\hbar+\frac{\alpha L_{p}^{2} \Delta p^{2}}{\hbar}\right],
$$

where $L_{p}$ denotes the Planck length. The GUP parameter $\alpha$ is typically considered to be a positive number of order unity in theoretical calculations. Note that GUP is largely heuristically "derived" from Gedanken-experiments and is often taken as a phenomenological model [1-4], though there exist attempts to make its formulation more mathematically rigorous, e.g. [5].

The generalized Hawking temperature for an asymptotically flat Schwarzschild black hole takes the form [6]

$$
T[\alpha>0]=\frac{M c^{2}}{4 \alpha \pi}\left(1-\sqrt{1-\frac{\alpha \hbar c}{G M^{2}}}\right) .
$$

The usual way to derive this expression is rather heuristic: the Heisenberg's uncertainty principle gives $\Delta p \sim \hbar / \Delta x$, also $E=k_{B} T$ and $E=p c$ (for massless particles), one arrives at the usual Hawking expression $c \Delta p / k \sim T \sim \hbar c^{3} /\left(2 k_{B} G M\right)$ by identifying $\Delta x \sim$ $r_{h} \equiv 2 G M / c^{2}$ as the uncertainty in the position of the emitted Hawking particle, which can be thought as the "quantum atmosphere" that extends some distance away from the horizon $r_{h}$ [7]. This is off by a factor $1 /(4 \pi)$ from the actual Hawking temperature. This "calibrating factor" is inserted by hand. One can perform the same calculation, using GUP in place of the usual uncertainty principle (and requiring that as $\alpha \rightarrow 0$ one recovers the latter, this fixes the sign in front of the square root term in eq. (1.2)).

A better way to understand eq. (1.2) is as follows: in various approaches to quantum gravity we know that the Bekenstein-Hawking entropy, $S$, of a black hole receives a logarithmic correction (see section 3 for more discussions). By the first law of black hole 
mechanics, $\mathrm{d} S=T \mathrm{~d} M$, one infers that Hawking temperature must be modified. It can be checked that eq. (1.2) is the right form that would give a logarithmic correction to the entropy. GUP can thus play the role of a phenomenological model that reproduces some known features of quantum gravity theories, thus allowing a simpler study of quantum gravitational effect without a full machinery of these theories.

Since $T[\alpha] \in \mathbb{R}$, such a GUP corrected black hole stops evaporating when it reaches mass $M=(\alpha \hbar c / G)^{1 / 2}=\sqrt{\alpha} M_{p}$, which is of the order of a Planck mass for $\alpha=O(1)$, resulting in a black hole remnant. Remnants [8] are problematic (see review [9]), but having a finite temperature object as an end state of Hawking evaporation is arguably more palatable than having a divergent temperature as in the usual picture of Hawking evaporation (assuming that $T \propto 1 / M$ holds for all time ${ }^{1}$ ). This remnant has finite temperature (which is quite high for $M \sim M_{p}$ ), but it is thermodynamically inert (its specific heat is zero, see also section 4) - i.e., it no longer interacts with the environment thermally. Instead, it behaves like an elementary particle, so the temperature here is better interpreted as the energy of the particle $\left(E=k_{B} T\right)$.

Nevertheless, GUP with positive $\alpha$ allows white dwarfs to be arbitrarily large $[14,15]$, which is observationally problematic. If one takes $\alpha<0$ however, the Chandrasekhar limit is restored, while at the same time there is no divergence in Hawking temperature at late time [16]. There is previously very little discussion on the possibility of negative $\alpha$, but see Scardigli-Casadio [17], in which $\alpha<0$ is derived by assuming that the GUP-corrected Hawking temperature can be obtained from Wick-rotating the effective Schwarzschild-like metric with

$$
g_{t t}=-\left(1-\frac{2 M}{r}+\varepsilon \frac{M^{2}}{r^{2}}\right)
$$

obtaining $\alpha=-4 \pi^{2} \varepsilon^{2}\left[M /\left(2 M_{p}\right)\right]^{2}<0$. See also [18], in which negative $\alpha$ was considered in the context of "sub-Planckian" black holes.

Note that if $\alpha<0$, gravity suppresses the uncertainty in the product $\Delta x \Delta p$. In fact there is a maximum momentum $\Delta p_{\max }$ such that $\Delta x \Delta p_{\max } \geqslant 0$. It thus seems that as Planck scale is approached, physics eventually becomes classical again. There are two possibilities:

(1) Planck scale physics is indeed not quantum [18]: this possibility has been considered in the literature, e.g., by taking $\hbar$ as a field, which goes to zero at high energy $[19,20]$, or by considering a lattice "world crystal" model [21]. Furthermore, if one fixes the mass scale, then in 4-dimensions, $G=\hbar c / M_{p}^{2}$ so that $\hbar \rightarrow 0$ can be viewed equivalently as $G \rightarrow 0$. Thus asymptotically safe gravity (see [22]) can also be considered as a similar scenario (see also [23] in the context of $f(R)$ gravity).

(2) GUP considered in this paper may not be exact, but instead only the first two terms of a series involving terms of higher powers in $\Delta p$ [24, 25]. For series expansion to be valid, $\Delta p$ cannot be too large.

\footnotetext{
${ }^{1}$ While this is commonly assumed, various approaches exist which modify the behavior at late time and thereby avoiding divergence in the temperature, e.g., by considering microcanonical ensembles [10-13].
} 


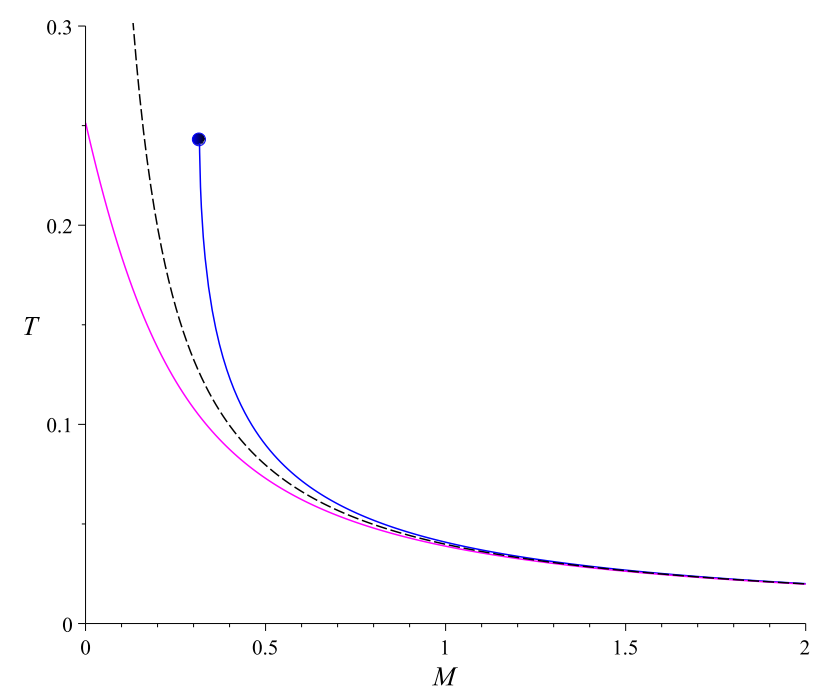

Figure 1. The various Hawking temperature of an asymptotically flat Schwarzschild black hole. The usual Hawking temperature corresponds to the middle dashed curve, which diverges as $M \rightarrow 0$. The infinity is "cured" with GUP correction: if $\alpha>0$, the temperature curve terminates at around $M \sim \sqrt{\alpha} M_{p}$, as shown by the right-most curve. If $\alpha<0$, GUP correction no longer imposes a lower bound on the black hole size. This corresponds to the left-most curve: the temperature remains finite as the black hole shrinks down to zero size.

For $\alpha<0$, we can re-write eq. (1.2) as

$$
T[\alpha<0]=-\frac{M c^{2}}{4|\alpha| \pi}\left(1-\sqrt{1+\frac{|\alpha| \hbar c}{G M^{2}}}\right) .
$$

Strictly speaking there is no remnant, since the mass of the black hole can go to zero when temperature reaches $T_{\max }=1 / \sqrt{4 \pi|\alpha|}$. See figure 1 . In other words, the black hole dissolves into particles of temperature $T_{\max }$. We shall refer to the $\alpha>0$ and $\alpha<0$ cases as, respectively, "positive correction" and "negative correction" to the uncertainty principle.

In [21], Jizba-Kleinert-Scardigli referred to the end state of negatively corrected GUP black hole as a "remnant with zero rest mass". As we shall see below, this turns out to be an apt description. In this work, we study the evolution of these black holes. We show that the black hole takes infinite amount of time to evaporate down to $M=0$, and so for all practical purposes it is a metastable, long-lived, remnant. From here onwards, we will set $G=c=\hbar=k_{B}=1$.

\section{The lifetime of GUP-corrected black holes}

The thermal evolution of an asymptotically flat Schwarzschild black hole is

$$
\frac{\mathrm{d} M}{\mathrm{~d} t}=-\alpha \sigma a T^{4}
$$

where $a$ is the radiation constant, $\sigma$ is essentially the area (in the geometric optics approximation, it is the photon capture cross section $\sigma=27 \pi M^{2}$ ), and $\alpha$ the greybody factor. For 


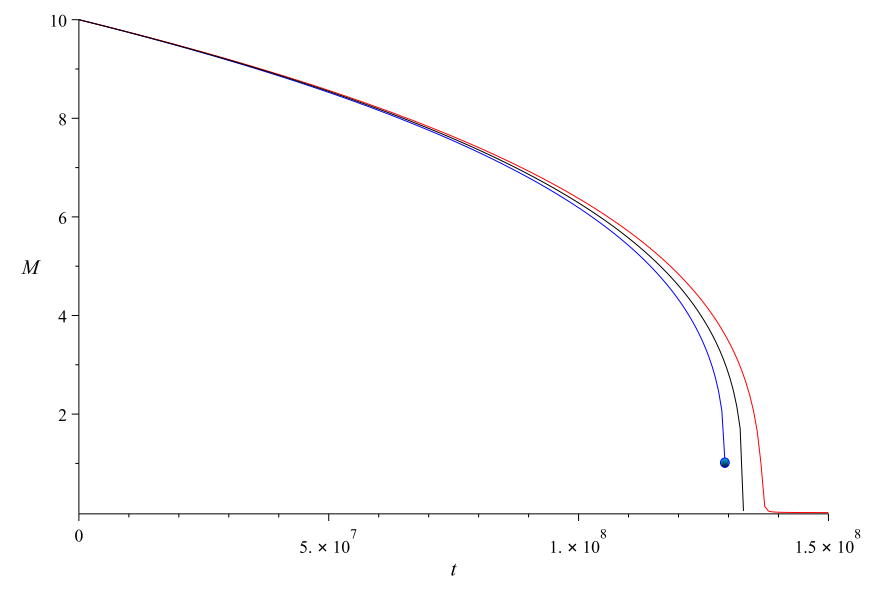

Figure 2. Mass evolution of Schwarzschild black holes with no GUP correction (black, middle curve), positive GUP correction (blue, left curve), and negative GUP correction (red, right curve). The positive GUP correction leads to a remnant in finite time, while negative GUP correction yields infinite lifetime. These contrast with the usual case without GUP correction, in which the black hole completely evaporates in finite time.

our purpose we are only interested in the general and qualitative behavior of the evolution, so we will simply consider the ODE

$$
\frac{\mathrm{d} M}{\mathrm{~d} t}=-\frac{1}{(8 \pi)^{4} M^{2}},
$$

since $\sigma \sim M^{2}$, and $T=1 /(8 \pi M)=T[\alpha=0]$. We retain the factor $8 \pi$ in the Hawking expression so as to be consistent with the GUP temperature expression (in the limit $\alpha \rightarrow 0$ ). It is well-known that Schwarzschild black hole completely evaporates in a finite time. In figure 2, we show the numerical plot (setting initial mass $M_{0}=10$ ). The black hole evaporates at about $t_{\text {evap }}=1.293 \times 10^{9}$. If we replace the temperature expression with eq. (1.2) instead, we have the GUP-modified ODE:

$$
\frac{\mathrm{d} M}{\mathrm{~d} t}=-M^{2} \cdot\left(\frac{M}{4 \alpha \pi}\right)^{4}\left(1-\sqrt{1-\frac{\alpha}{M^{2}}}\right)^{4} .
$$

We find that a positively corrected GUP black hole with $\alpha=1$ stops evaporating at a time somewhat less than $t_{\text {evap }}$, but of the same order of magnitude. Our main interest is to investigate what happens when $\alpha<0$. Take, e.g., $\alpha=-1$. The result is intriguing: the black hole evaporation is divided into two regimes: in the first stage, it follows the same qualitative behavior as that of $\alpha=0$ and $\alpha>0$ black holes. However, as it approaches the horizontal axis, it turns around and asymptotes along the $t$-axis. In fact, it never reaches zero mass. See figure 2. Decreasing $\alpha$ (i.e. more negative) has the effect of pushing the curve towards the right, as shown in figure 3.

To appreciate the infinite lifetime of such black holes, we note that for negative $\alpha$, the mass evolution follows

$$
\frac{\mathrm{d} M}{\mathrm{~d} t}=\frac{M^{6}}{(4|\alpha| \pi)^{4}}\left(1-\sqrt{1+\frac{|\alpha|}{M^{2}}}\right)^{4} .
$$




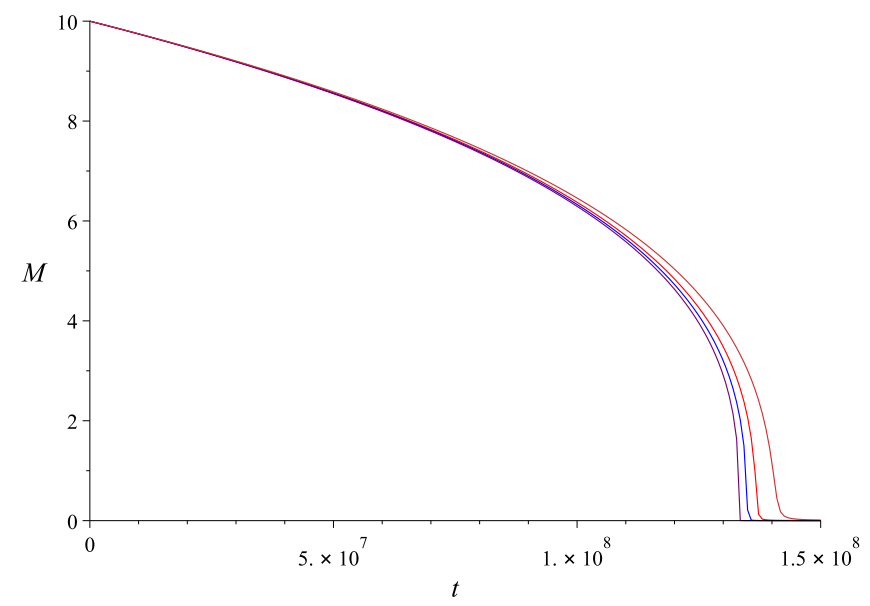

Figure 3. Mass evolution of Schwarzschild black holes with negative GUP corrections. From right to left, the curves correspond to GUP parameters $\alpha=-2,-1,-0.5,-0.1$, respectively. Decreasing $\alpha$ (i.e. more negative) has the effect of pushing the curve towards the right. They all asymptote towards zero.

When $M$ has become sufficiently small, we have

$$
\frac{\mathrm{d} M}{\mathrm{~d} t} \sim-\frac{M^{2}}{(4 \pi)^{4} \alpha^{2}}
$$

This has solution of the form

$$
M=M_{0}\left(\frac{256 \pi^{4} \alpha^{2}}{256 \pi^{4} \alpha^{2}+M_{0} t}\right)
$$

where $M_{0}$ is the "initial" (small) mass. Clearly $M \rightarrow 0$ as $t \rightarrow \infty$.

\section{Sparsity makes lifetime even longer}

Hawking temperature is unlike a typical blackbody: the wavelength of a Hawking particle (without GUP correction) is [7] $\lambda=2 \pi / T=16 \pi^{2} M \approx 79 r_{h} \gg r_{h}$. This is in contrast with a typical blackbody radiation, which has wavelength much smaller than the size of the emitting body. For black holes, the emitting surface is taken to be the geometric optics cross section $\sigma$. One can look at the dimensionless ratio $^{2} \eta:=\lambda^{2} / \sigma$.

If $\eta \ll 1$, then we have a typical blackbody, which emits continuously. On the other hand, $\eta \gg 1$ means the Hawking radiation is sparse: a particle is randomly emitted in a discrete manner, with "pauses" in between. (Such emission also contributes to the random walk of black hole, as the result of backreaction from Hawking emission [26].) See [27-29] for detailed discussions.

A recent study investigated the effect of GUP with positive $\alpha$ on the sparsity of Hawking radiation, and found that the radiation is no longer quite sparse near the Planck scale [30]. We will re-do the calculations as there are some minor disagreements with [30],

\footnotetext{
${ }^{2}$ We have omitted a numerical prefactor in the definition of $\eta$, which plays no role in our discussion below.
} 


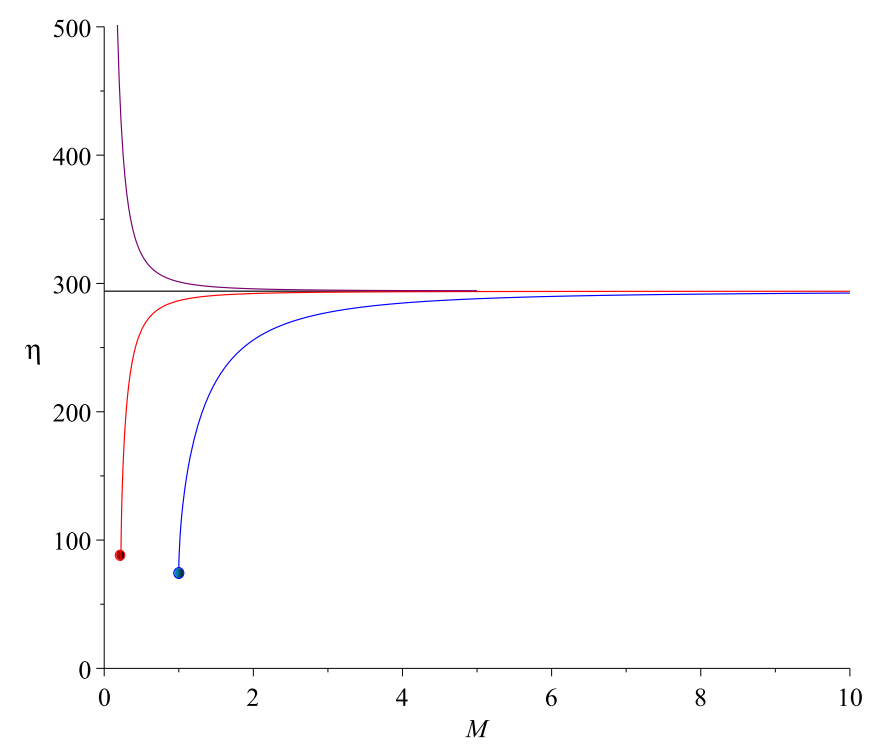

Figure 4. The sparsity of Hawking radiation. The constant black line corresponds to the usual Hawking evaporation - the radiation remains sparse even towards the end of the evaporation. The bottom two curves are for $\alpha=1$ (bottom right curve, blue) and $\alpha=0.05$ (bottom left, red), respectively. For $\alpha>0$ : GUP correction leads to the decrease in $\eta$, and the radiation becomes less sparse towards the end. However, for $\alpha<0$, we get an ever-increasing $\eta$, so the radiation becomes extremely sparse. Shown here (top, purple), is an example for which $\alpha=-0.05$.

which nevertheless do not affect their results. We then consider the case of negative $\alpha$, and found that it enhances the sparsity. In fact, the radiation becomes infinitely sparse. Eq. (2.2) does not take into account the effect of sparsity, instead mass loss is only treated as particle mass loss associated to a given temperature [27-29]. Once the wave effect of the radiation is taken into account, sparsity becomes a crucial feature that extends the black hole lifetime. For our negatively corrected GUP black hole, its lifetime is already infinite before taking sparsity into account. In addition, we have not taken into account greybody factors, which would suppress the rate even further. Therefore it is safe to conclude that these black holes do have infinite lifetime (unless new physics comes into effect). Now let us look at the details of the calculations.

After GUP correction (with $\alpha$ of either signs), we have $\lambda[\alpha]=2 \pi / T[\alpha]$. Thus, GUP corrected sparsity is

$$
\eta[\alpha]:=\frac{\lambda^{2}[\alpha]}{\sigma}=\frac{\lambda^{2}[\alpha]}{27 \pi M^{2}} .
$$

In [30], the authors considered the fact that $\sigma=(27 / 4) \pi r_{h}^{2}$. Denoting $A=\pi r_{h}^{2}$, they considered a GUP-correction to $A$ essentially via the fact that the entropy (and thus the area) is modified under GUP correction via the first law of black hole mechanics to (up to a constant):

$$
S[\alpha]=2 \pi\left[M^{2}+M \sqrt{M^{2}-\alpha}-\alpha \ln \left(M+\sqrt{M^{2}-\alpha}\right)\right] .
$$

Note that there is a logarithmic term correction to the entropy. However, in the derivation of the GUP-Hawking temperature itself, it is usually considered that the horizon 
is still at $r=2 M \sim \Delta x$, so that to be self-consistent, the horizon size should not receive any GUP correction. ${ }^{3}$ As a consequence, the area law $S=A / 4$ does not hold. ${ }^{4}$ Hence, we choose to not consider correction to the horizon size (for the same reason, the $\sigma$ in eq. (2.3) remains just $\sigma \sim M^{2}$, this agrees with [33,34]). Despite this difference, the result for $\alpha>0$ remains qualitatively the same as [30], namely $\eta$ decreases as the black hole mass gets near the Planckian regime, ${ }^{5}$ see figure 4 . Another small difference with [30] is a technical one: they have used series expansion in the temperature, while we retained the full expression. This is why their black hole mass can become zero, while we know that for $\alpha>0$ there should be a minimum mass corresponds to the remnant mass.

Interestingly, as shown in figure 4 , for $\alpha<0$, the sparsity increases without bound. It is easy to see that it diverges: $M$ can tend to zero for $\alpha<0$, while $T$ tends to a constant value $1 / \sqrt{4 \pi|\alpha|}$, so from eq. (3.1), $\eta \rightarrow \infty$.

\section{Discussion: the final fate of GUP-corrected black holes}

A common belief is that black holes should completely disappear at the end of Hawking evaporation, since there are serious problems with the remnant proposal, as discussed in the review paper [9]. Nevertheless, though unpopular, remnant still deserves a closer scrutiny.

There are many reasons to suspect that the thermal description of black hole, eq. (2.1), cannot hold for all time. In addition to micro-canonical ensemble considerations [1013], from quantum information point of view, it is widely believed that unitarity requires information to "leak" out of the black hole after Page time [36, 37] (though is highly scrambled among the quantum entangled Hawking particles) about half-way through the evaporation. Subtle quantum effects might affect the evolution of black holes. Even without these effects, as the black hole becomes hotter and hotter, it is likely that new physics would come into effect. The evolution of black holes might then get modified. In other words, perhaps quantum gravity will resolve the divergence in the temperature, much like how it might resolve black hole singularities.

GUP is a quantum gravity inspired phenomenological model that could accommodate minimal length (for positive GUP parameter $\alpha$ ) and black hole remnant, see review [38], so it is interesting to investigate how black holes evolve under GUP-corrected Hawking process. In this work, we explored the possibility that $\alpha<0$, which is much less studied. Despite the absence of lower bound for the mass, complete evaporation cannot be achieved in finite time. In addition, sparsity of the Hawking radiation becomes infinite as $M \rightarrow 0$. Considerations of greybody factors will likely "prolong" the (already infinite) lifetime. The end state of the black hole should be viewed as a "zero mass remnant" - in the sense

\footnotetext{
${ }^{3}$ More explicitly, in the heuristic derivation of Hawking temperature via the usual Heisenberg's uncertainty principle, one takes $\Delta x \sim 2 M$, and $T \sim \Delta p \sim 1 / \Delta x$. In the GUP case, one replaces $\Delta p$ with the GUP corrected version, but $\Delta x$ remains $2 M[6]$.

${ }^{4}$ This is consistent with other approaches of quantum gravity in which logarithmic correction appears in the entropy expression, but one usually does not modify the area expression, e.g. [31, 32].

${ }^{5}$ We note that a previous study without utilizing GUP, but taking into account backreaction instead, leads to the same conclusion [35], including the existence of a remnant.
} 
that it is a metastable state that asymptotes to zero mass. (A similar result was obtained in [39] via higher curvature corrections to the Hilbert-Einstein action.)

Lastly we remark on the heat capacity,

$$
C:=\frac{\mathrm{d} M}{\mathrm{~d} T}=\frac{\mathrm{d} M}{\mathrm{~d} t}\left(\frac{\mathrm{d} T}{\mathrm{~d} t}\right)^{-1} .
$$

It can be shown that $C[\alpha] \leqslant 0 \forall \alpha \in \mathbb{R}$, just like the usual Schwarzschild black hole. However, for $\alpha>0$, as the remnant mass is approached, $C \rightarrow 0$. Thus, a remnant has zero heat capacity - it is thermodynamically inert, as explained in section 1. For $\alpha<0$ however, heat capacity is always negative, so the metastable remnant interacts thermally with the environment, as pointed out in [21].

It is interesting how different signs of $\alpha$ lead to black hole remnants: for $\alpha>0$, it is a stable remnant of finite "temperature" (best interpreted as the energy of the remnant "particle", see section 1); whereas for $\alpha<0$, it is a metastable, long-lived remnant that approaches zero rest mass asymptotically. The latter cannot be directly inferred from the $T-M$ plot alone (figure 1), one must instead study its evolution equation and sparsity.

The implications for such a remnant deserve a closer study. We speculate on some possibilities: firstly, remnants could help to resolve the information paradox (see [9]). It was further proposed that the sparsity in Hawking radiation could encode information between the emission gap without disturbing the time-averaged emission spectrum [27]. Although this is unlikely to be the full resolution to the paradox, it would be interesting to investigate in details the quantum information aspects of the Hawking particles emitted by our long-lived remnant with infinite sparsity towards the end of time.

Secondly, black hole remnants, including those obtained from positively corrected GUP [33, 34], had been considered as candidate of dark matter — see, e.g., [40] and the various references in [41]. Remnants as dark matter is still allowed by observational constraints [42]. One could look into how phenomenologically different the zero mass remnant might be as dark matter candidate, compared to the $\alpha>0$ remnant.

\section{Acknowledgments}

YCO thanks the National Natural Science Foundation of China (grant No.11705162) and the Natural Science Foundation of Jiangsu Province (No.BK20170479) for funding support. He also acknowledges the China Postdoctoral Science Foundation (grant No.17Z102060070) for partial support. YCO thanks Brett McInnes for comments and suggestions.

Open Access. This article is distributed under the terms of the Creative Commons Attribution License (CC-BY 4.0), which permits any use, distribution and reproduction in any medium, provided the original author(s) and source are credited.

\section{References}

[1] M. Maggiore, A generalized uncertainty principle in quantum gravity, Phys. Lett. B 304 (1993) 65 [hep-th/9301067] [INSPIRE]. 
[2] M. Maggiore, Quantum groups, gravity and the generalized uncertainty principle, Phys. Rev. D 49 (1994) 5182 [hep-th/9305163] [INSPIRE].

[3] F. Scardigli, Generalized uncertainty principle in quantum gravity from micro-black hole Gedanken experiment, Phys. Lett. B 452 (1999) 39 [hep-th/9904025] [INSPIRE].

[4] R.J. Adler and D.I. Santiago, On gravity and the uncertainty principle, Mod. Phys. Lett. A 14 (1999) 1371 [gr-qc/9904026] [INSPIRE].

[5] M. Isi, J. Mureika and P. Nicolini, Self-completeness and the generalized uncertainty principle, JHEP 11 (2013) 139 [arXiv:1310.8153] [INSPIRE].

[6] R.J. Adler, P. Chen and D.I. Santiago, The generalized uncertainty principle and black hole remnants, Gen. Rel. Grav. 33 (2001) 2101 [gr-qc/0106080] [INSPIRE].

[7] S.B. Giddings, Hawking radiation, the Stefan-Boltzmann law and unitarization, Phys. Lett. B 754 (2016) 39 [arXiv: 1511.08221] [INSPIRE].

[8] Y. Aharonov, A. Casher and S. Nussinov, The unitarity puzzle and Planck mass stable particles, Phys. Lett. B 191 (1987) 51 [INSPIRE].

[9] P. Chen, Y.C. Ong and D.-H. Yeom, Black hole remnants and the information loss paradox, Phys. Rept. 603 (2015) 1 [arXiv: 1412.8366] [InSPIRE].

[10] R. Casadio, B. Harms and Y. Leblanc, Microfield dynamics of black holes, Phys. Rev. D 58 (1998) 044014 [gr-qc/9712017] [INSPIRE].

[11] R. Casadio and B. Harms, Black hole evaporation and large extra dimensions, Phys. Lett. B 487 (2000) 209 [hep-th/0004004] [INSPIRE].

[12] S. Hossenfelder, What black holes can teach us, in Focus on black hole research, Nova Science Publishers, U.S.A., (2005), pg. 155 [hep-ph/0412265] [INSPIRE].

[13] R. Casadio and B. Harms, Microcanonical description of (micro) black holes, Entropy 13 (2011) 502 [arXiv:1101.1384] [INSPIRE].

[14] R. Rashidi, Generalized uncertainty principle and the maximum mass of ideal white dwarfs, Annals Phys. 374 (2016) 434 [arXiv: 1512.06356] [INSPIRE].

[15] M. Moussa, Effect of generalized uncertainty principle on main-sequence stars and white dwarfs, Adv. High Energy Phys. 2015 (2015) 343284 [arXiv: 1512.04337] [INSPIRE].

[16] Y.C. Ong, Generalized uncertainty principle, black holes and white dwarfs: a tale of two infinities, JCAP 09 (2018) 015 [arXiv:1804.05176] [INSPIRE].

[17] F. Scardigli and R. Casadio, Gravitational tests of the generalized uncertainty principle, Eur. Phys. J. C 75 (2015) 425 [arXiv: 1407.0113] [InSPIRE].

[18] B.J. Carr, J. Mureika and P. Nicolini, Sub-Planckian black holes and the generalized uncertainty principle, JHEP 07 (2015) 052 [arXiv: 1504.07637] [INSPIRE].

[19] S. Hossenfelder, Gravity can be neither classical nor quantized, in The frontiers collection, Springer International Publishing, Cham, Switzerland, (2015), pg. 219 [arXiv:1212.0454] [INSPIRE].

[20] S. Hossenfelder, A possibility to solve the problems with quantizing gravity, Phys. Lett. B $\mathbf{7 2 5}$ (2013) 473 [arXiv:1208.5874] [INSPIRE].

[21] P. Jizba, H. Kleinert and F. Scardigli, Uncertainty relation on world crystal and its applications to micro black holes, Phys. Rev. D 81 (2010) 084030 [arXiv:0912.2253] [INSPIRE].

[22] M. Niedermaier, The asymptotic safety scenario in quantum gravity: an introduction, Class. Quant. Grav. 24 (2007) R171 [gr-qc/0610018] [INSPIRE]. 
[23] B. Greene, K. Hinterbichler, S. Judes and M.K. Parikh, Smooth initial conditions from weak gravity, Phys. Lett. B 697 (2011) 178 [arXiv:0911.0693] [InSPIRE].

[24] G. Amelino-Camelia, M. Arzano, Y. Ling and G. Mandanici, Black-hole thermodynamics with modified dispersion relations and generalized uncertainty principles, Class. Quant. Grav. 23 (2006) 2585 [gr-qc/0506110] [INSPIRE].

[25] S. Das and E.C. Vagenas, Universality of quantum gravity corrections, Phys. Rev. Lett. 101 (2008) 221301 [arXiv:0810.5333] [INSPIRE].

[26] D.N. Page, Is black hole evaporation predictable?, Phys. Rev. Lett. 44 (1980) 301 [INSPIRE].

[27] F. Gray, S. Schuster, A. Van-Brunt and M. Visser, The Hawking cascade from a black hole is extremely sparse, Class. Quant. Grav. 33 (2016) 115003 [arXiv:1506.03975] [inSPIRE].

[28] M. Visser, F. Gray, S. Schuster and A. Van-Brunt, Sparsity of the Hawking flux, in Proceedings, $14^{\text {th }}$ Marcel Grossmann Meeting on Recent Developments in Theoretical and Experimental General Relativity, Astrophysics and Relativistic Field Theories (MG14), vol. 2, Rome, Italy, 12-18 July 2015, World Scientific, Singapore, (2017), pg. 1724 [arXiv: 1512.05809] [INSPIRE].

[29] W. Mück, Hawking radiation is corpuscular, Eur. Phys. J. C 76 (2016) 374 [arXiv: 1606.01790] [INSPIRE].

[30] A. Alonso-Serrano, M.P. Dąbrowski and H. Gohar, Generalized uncertainty principle impact onto the black holes information flux and the sparsity of Hawking radiation, Phys. Rev. D 97 (2018) 044029 [arXiv: 1801.09660] [INSPIRE].

[31] A. Ghosh and P. Mitra, A bound on the log correction to the black hole area law, Phys. Rev. D 71 (2005) 027502 [gr-qc/0401070] [INSPIRE].

[32] P. Chen and C.-H. Wang, Where is $\bar{h}$ hiding in entropic gravity?, arXiv:1112.3078 [INSPIRE].

[33] P. Chen and R.J. Adler, Black hole remnants and dark matter, Nucl. Phys. Proc. Suppl. 124 (2003) 103 [gr-qc/0205106] [INSPIRE].

[34] P. Chen, Inflation induced Planck-size black hole remnants as dark matter, New Astron. Rev. 49 (2005) 233 [astro-ph/0406514] [INSPIRE].

[35] A. Paul and B.R. Majhi, Hawking evaporation cascade in the presence of backreaction effect, Int. J. Mod. Phys. A 32 (2017) 1750088 [arXiv:1601.07310] [InSPIRE].

[36] D.N. Page, Average entropy of a subsystem, Phys. Rev. Lett. 71 (1993) 1291 [gr-qc/9305007] [INSPIRE].

[37] D.N. Page, Time dependence of Hawking radiation entropy, JCAP 09 (2013) 028 [arXiv: 1301.4995] [INSPIRE].

[38] S. Hossenfelder, Minimal length scale scenarios for quantum gravity, Living Rev. Rel. 16 (2013) 2 [arXiv: 1203.6191] [INSPIRE].

[39] P. Bueno and P.A. Cano, Universal black hole stability in four dimensions, Phys. Rev. D 96 (2017) 024034 [arXiv: 1704.02967] [INSPIRE].

[40] K. Nozari and S.H. Mehdipour, Gravitational uncertainty and black hole remnants, Mod. Phys. Lett. A 20 (2005) 2937 [arXiv:0809.3144] [INSPIRE].

[41] C. Rovelli and F. Vidotto, Small black/white hole stability and dark matter, arXiv: 1805.03872 [INSPIRE].

[42] B. Carr, F. Kühnel and M. Sandstad, Primordial black holes as dark matter, Phys. Rev. D 94 (2016) 083504 [arXiv: 1607.06077] [INSPIRE]. 